\title{
BMJ Global Health Proactive community case management and child survival in periurban Mali
}

To cite: Johnson AD, Thiero 0 , Whidden C, et al. Proactive community case management and child survival in periurban Mali. BMJ Glob Health 2018;3:e000634. doi:10.1136/ bmjgh-2017-000634

Handling editor Seye Abimbola

- Additional material is published online only. To view, please visit the journal online (http://dx.doi.org/10.1136/ bmjgh-2017-000634).

Received 6 November 2017 Revised 7 February 2018 Accepted 8 February 2018
Check for updates

${ }^{1}$ Department of Medicine, University of California, San Francisco, School of Medicine, San Francisco, California, USA

${ }^{2}$ Muso, Bamako, Mali, San Francisco, California, USA ${ }^{3}$ Tulane University, School of Public Health and Tropical Medicine, New Orleans, Louisiana, USA

${ }^{4}$ Malaria Research and Training Centre, Faculty of Medicine and Odontostomatology, University of Sciences, Techniques and Technologies of Bamako, Bamako, Mali

${ }^{5}$ Ministry of Public Health and Hygiene, Bamako, Mali

Correspondence to

Dr Ari D Johnson;

ari.johnson@ucsf.edu

\section{ABSTRACT}

The majority of the world's population lives in urban areas, and regions with the highest under-five mortality rates are urbanising rapidly. This 7-year interrupted time series study measured early access to care and under-five mortality over the course of a proactive community case management (ProCCM) intervention in periurban Mali. Using a cluster-based, population-weighted sampling methodology, we conducted independent cross-sectional household surveys at baseline and at 12, 24, 36, 48, 60,72 and 84 months later in the intervention area. The ProCCM intervention had five key components: (1) active case detection by community health workers (CHWs), (2) CHW doorstep care, (3) monthly dedicated supervision for CHWs, (4) removal of user fees and (5) primary care infrastructure improvements and staff capacity building. Under-five mortality rate was calculated using a Cox proportional hazard survival regression. We measured the percentage of children initiating effective antimalarial treatment within 24 hours of symptom onset and the percentage of children reported to be febrile within the previous 2 weeks. During the intervention, the rate of early effective antimalarial treatment of children 0-59 months more than doubled, from $14.7 \%$ in 2008 to $35.3 \%$ in 2015 (OR 3.198, $\mathrm{P}<0.0001$ ). The prevalence of febrile illness among children under 5 years declined after 7 years of the intervention from $39.7 \%$ at baseline to $22.6 \%$ in 2015 (OR 0.448, $\mathrm{P}<0.0001$ ). Communities where ProCCM was implemented have achieved an under-five mortality rate at or below 28/1000 for the past 6 years. In 2015, underfive mortality was $7 / 1000$ (HR $0.039, \mathrm{P}<0.0001$ ). Further research is needed to elucidate the mechanisms of action and generalizability of ProCCM.

\section{INTRODUCTION}

The majority of the world's population now lives in urban areas. ${ }^{1}$ While Africa remains predominantly rural, the continent is urbanising faster than other regions and is projected to become $56 \%$ urban by $2050 .{ }^{1}$ With more than half of sub-Saharan Africa's urban population living in slums and informal settlements, ${ }^{2}$ this demographic shift has major implications for health and health equity. Characterised by extreme poverty, environmental degradation and a lack of basic infrastructure, these urban areas are vulnerable to

\section{Summary box}

What is already known about this topic?

- Countries with a high burden of under-five mortality are urbanising rapidly.

- Community health workers (CHWs) are being deployed across countries in sub-Saharan Africa to reduce under-five mortality through integrated community case management (iCCM), predominantly in rural contexts.

- National efforts to deploy CHWs have confronted significant challenges, and the expected benefits of iCCM have not been found in all contexts.

\section{What are the new findings?}

- Seven years following the launch of proactive community case management (ProCCM), the areas of the intervention had an under-five mortality rate of $7 / 1000$.

- Communities served by ProCCM have sustained a rate of under-five mortality lower than any country in sub-Saharan Africa.

Recommendations for policy

- Facing rapid urbanisation, health systems will need to overcome multiple barriers urban patients face to accessing timely care in order to stop preventable child deaths.

- Further research is urgently needed on the core strategies of ProCCM to determine their generalizability and mechanisms of action in improving child mortality.

infectious diseases such as malaria, diarrhoea and pneumonia, to new pandemic outbreaks and to an emerging non-communicable disease burden.

Malaria, diarrhoea, pneumonia and neonatal illness, diseases with evidence-based and cost-effective prevention and treatment methods, ${ }^{3-5}$ constitute the leading causes of childhood death in sub-Saharan Africa. ${ }^{6}$ Timely access to care is a key element in the management of these diseases, which progress rapidly. Delayed access to effective care has been correlated with increased under-five 
mortality from malaria, ${ }^{78}$ diarrhoeal disease, ${ }^{9}$ acute respiratory infection ${ }^{1011}$ and neonatal asphyxia. ${ }^{12-14}$

Weak and poorly designed health systems create barriers to timely access to care. ${ }^{1415}$ Fees at point of care are associated with decreased and delayed utilisation of care, and removing user fees correlates with increases in healthcare utilisation, particularly among the poor. ${ }^{16-20}$ Inadequate human resources for health, gender inequality, inadequate infrastructure and poverty also impede access to care ${ }^{1421-26}$ Geographical distance, though a more prominent barrier in rural contexts, can still impede access to care for the urban poor. ${ }^{21}$ As a result, facility-based services alone, in both rural and urban contexts, frequently do not provide adequate, timely and equitable access to care. ${ }^{27}$

Community case management (CCM) removes barriers to access by training and supporting community health workers (CHWs) to diagnose, treat and refer sick children in the communities in which they live. Since 2004, WHO and Unicef have issued joint statements promoting CCM of malaria, acute diarrhoea, pneumonia, acute malnutrition and newborn survival. These statements are based on a body of evidence showing CCM to be effective in increasing care seeking, reducing treatment failure rates and reducing mortality due to malaria, diarrhoea, pneumonia and all-cause mortality. ${ }^{28-36}$ In 2012, these community-based services were compiled into a package of care known as integrated community case management (iCCM) of childhood illnesses. ${ }^{27}$

Twenty-eight countries in sub-Saharan Africa have adopted ICCM as a strategy to improve child health and survival. ${ }^{37}$ iCCM programmes differ in many ways, including CHW workflow, recruitment, training, health systems integration, supervision and compensation. ${ }^{37-39}$ However, one particular pattern emerges among iCCM policies and plans in sub-Saharan Africa. In Mali, Liberia, Ghana, Burkina Faso, Ethiopia, Malawi and other countries, iCCM has been used as a strategy to improve child health primarily in remote rural settings. As the sub-Saharan African region urbanises rapidly, further research is needed urgently on the feasibility and effects of iCCM on improving access to care, morbidity and mortality in urban areas.

National efforts to scale iCCM have confronted significant challenges, and the expected benefits of iCCM have not been found in all contexts. ${ }^{40-42}$ Three recent independent evaluations of national iCCM programmes in Burkina Faso, Ethiopia and Malawi found no significant impact on care seeking or child mortality. ${ }^{43-46}$ These results emphasise the importance of testing models for improving iCCM service delivery and the potential impact of improved models in both urban and rural contexts.

Geographical proximity to a health centre does not ensure access to services, as many health system barriers, including direct and indirect costs of care, availability of services, shortage and insufficient training of staff and gender inequality, persist in urban contexts. ${ }^{478} \mathrm{CHWs}$ do more than extend the health system into geographically remote areas; they may be important for overcoming barriers to care that cut across rural-urban divides.

However, research and programmatic effort for iCCM and CHW services have been considerably more limited in urban settings than in rural communities. CHWs have been shown to improve rates of exclusive breastfeeding, infant weight and care seeking through prenatal and postnatal home visits in a periurban settlement in South Africa. ${ }^{40}$ The BRAC Manoshi programme significantly reduced home births, maternal mortality and neonatal mortality through CHW home visits in urban slums in Bangladesh. ${ }^{51}$ Pilot projects in five African cities suggest that CCM of malaria is relevant in urban settings, where community-based agents were often the first point of care for febrile children, and most children were treated within 24 hours of symptom onset. ${ }^{5253}$

As Mali expands iCCM to remote rural villages, the government and its partners have expressed interest in testing alternative models of iCCM delivery and its potential impact in urban areas. Proactive community case management (ProCCM), a model for integrating iCCM into health systems at scale, is being implemented and tested in periurban and rural Mali. ProCCM takes into account the multiplicity of barriers patients face when trying to obtain care, focusing on early access. ProCCM CHWs conduct daily door-to-door proactive case detection, searching for and identifying patients who need care. For all patients identified, CHWs provide free-ofcharge doorstep evaluation, diagnostics, treatment, referral, counselling and follow-up.

In 2013, an interrupted time series study documented changes in early access to care and under-five mortality in the periurban area of Yirimadio, Mali after the roll-out of a health system strengthening intervention that included ProCCM of malaria. Three years after the introduction of the intervention, early access to care increased nearly twofold ( $\mathrm{PR}=1.89, \mathrm{P}=0.0195)$, and the total number of patient-provider contacts increased 10-fold. Under-five mortality was 155 per 1000 live births at baseline and 17 per 1000 live births at year three (HR 0.10, $\mathrm{P}<0.0001){ }^{54}$

In Mali and across sub-Saharan Africa, iCCM is being designed for and implemented primarily in remote rural settings with mixed results. Testing alternative models of delivery of iCCM services and their potential impact in both rural and urban contexts is, therefore, important. Here, we present a 7-year follow-on interrupted time series analysis assessing whether the changes in access to care and child mortality seen in ProCCM communities have been sustained over time.

\section{SETTING}

Mali has the world's sixth highest national under-five child mortality rate, estimated at 114 deaths/1000 live births in $2015 .^{55}$ The public sector health system has used a user fee-for-service healthcare financing model since the Bamako Initiative of $1987 .^{56}$ Covering an area of approximately 3.5 square miles on the edge of Bamako, 
the periurban area of Yirimadio has experienced rapid population growth. Based on household census enumerations by Muso, the population in the study area was 56371 in 2011 and 77132 in 2013. Several factors contributed to the rapid population growth of the area. These include in-migration from rural areas, out-migration from the city's centre and Mali's high fertility ratefactors consistent with trends of urban growth across the region. ${ }^{57}$ Certain patients also reported that they had moved to the area to seek care in the context of a system that provided access to healthcare without fees. During the 2012 security crisis in Mali, residents from Mali's north were displaced and sought refuge in the country's south, including the areas of the study.

At baseline, the public sector provided primary care through a community health centre that consisted of a consultation room, medicine dispensary, observation room and two rooms for labour and delivery. Local volunteers, known as relais, were trained by the Ministry of Health to share key maternal and child health messages with other community members. At baseline, there were no CHWs providing community-based management of childhood illness.

\section{DESCRIPTION OF THE PROCCM INTERVENTION}

The non-governmental organisation Muso ${ }^{58}$ and the Malian Ministry of Health and Public Hygiene jointly launched ProCCM in Yirimadio in September 2008, designed to overcome health system barriers to timely access to preventive and curative care.

ProCCM involved five key components: (1) proactive case detection: CHWs conducted proactive case detection, searching for patients door-to-door for at least 2 hours per day, 6 days per week; (2) doorstep care: CHWs provided counselling, diagnosis, treatment and referral according to standard iCCM protocols ${ }^{27}$; (3) dedicated supervision: CHWs received monthly individual supervision sessions and weekly group supervision sessions from a dedicated CHW supervisor; (4) user fee removal: no user fees were charged for community-based care. User fees were also removed at the Ministry of Health primary care centre for all patients who could not afford to pay, as determined by the patient self-reporting to his or her CHW and (5) primary care centre improvements: primary care capacity building for the Ministry of Health primary care centre, through infrastructure expansion and staff training.

Proactive CHWs providing doorstep care, removal of user fees and health centre construction were all fully deployed by September 2008. In the context of rapid population growth, the number of CHWs increased to target a CHW: population ratio of 1:1000, from $20 \mathrm{CHWs}$ in 2008 to 75 in 2013 and then to 150 in 2015, of which $95 \%$ were women. Group supervision visits occurred weekly, held by a supervising programme manager from 2008 to 2013 and then by a dedicated cadre of CHW supervisors from 2013 to 2015. Individual monthly field supervision visits from a dedicated cadre of CHW supervisors began in July 2013. To keep up with population growth, additional health centre construction further expanded the public sector primary health centre in 2009, 2010 and 2013.

CHWs received 36 days of foundational training. The first CHWs deployed in 2008 received approximately half of this training preservice and the rest in modular fashion as in-service training. For CHWs subsequently recruited, most of this training was shifted to be preservice, with weekly group supervision meetings serving to review and reinforce key skills and competencies. CHWs were instructed and supervised to conduct at least 2 hours of proactive case detection home visits per day, 6 days per week, with a goal of visiting each household at least twice per month. CHWs maintained the autonomy to determine the details of their own workflow, including what times of day they conducted their visits and what order or pattern they visited homes. This high level of CHW autonomy was by design, positioning the CHWs as leaders in assessing the needs of their communities, adapting to local context, triaging urgent cases and adjusting their workflows to meet their goals.

Outside their proactive case detection activities, CHWs were instructed to be available on call at all times to patients who sought their services. In addition to proactive case detection for patients of all ages, CHWs provided a package of services consistent with iCCM clinical protocols, including: (1) counselling; (2) diagnosis of malaria (all ages), pneumonia, diarrhoeal disease and malnutrition for children under five; (3) treatment of uncomplicated cases of malaria, pneumonia, diarrhoeal disease and moderate acute malnutrition for children under five; (4) referral and accompaniment of patients with danger signs or conditions outside the CHW's scope of practice to the primary health centre-this included identification and evacuation of patients with danger signs in the neonatal period, such as lethargy and poor feeding; (5) follow-up visits to support adherence and monitor response to therapy at 24 hours, 48 hours and 72 hours for all sick patients after the initial evaluation, with an additional visit after 5 days for patients with diarrhoeal disease and (6) active case detection and evacuation of sick newborns, pregnant women and postpartum women to the primary care facility for treatment.

CHWs were recruited from the area of the intervention. Selection criteria included an ability to read and write in the local language (Bamanankan), communication skills, a commitment to community health, work ethic and an age between 18 and 45 years old. Female candidates were encouraged to apply. For neighbourhoods without a qualified candidate, the nearest qualified candidate was selected. CHWs were compensated for their work with a monthly payment of FCFA40 000 (approximately US\$75). Each CHW supervisor was paid a starting salary of FCFA100 000 (approximately US\$188) per month and was responsible for 15-20 CHWs. 


\section{CONCURRENT INTERVENTIONS IN THE STUDY AREA}

During the period of the study, other interventions were introduced in the study area that sought to address disparities in the socioeconomic determinants of health, through microenterprise support, education and community organising. A non-formal education programme, developed by the organisation Tostan,${ }^{59}$ trained 1032 adults and adolescents in the years 2009-2012 with a curriculum that included human rights, problem solving, project management, democracy, health, hygiene, literacy, numeracy and income generation skills. Also, during the period of 2009-2012, 238 community organisers were trained to identify and implement community-designed health and development projects, including planting trees and mobilising community members around vaccination campaigns. From 2008 to 2015, a Muso microenterprise programme with 477 participants provided training, savings structures, grants and/or loans to women to start or expand revenue-generating activities.

In addition, during the period of the study, the Malian Ministry of Health and Public Hygiene adopted a national strategy targeting universal coverage with long-lasting insecticide-treated nets (LLINs) for malaria prevention. Distribution campaigns of LLINs were undertaken by Muso, Population Services International (PSI) and the Malian Ministry of Health and Public Hygiene in the study area in 2011 and 2015.

\section{PARTICIPANTS AND SAMPLING METHODOLOGY}

We conducted an adequacy evaluation based on a full coverage intervention to measure changes in early access and under-five mortality outcomes in the surveyed area. ${ }^{60}$ Starting in 2008, we conducted independent, cross-sectional household surveys in the intervention area at baseline and at 12, 24, 36, 48, 60, 72 and 84 months subsequently. The surveys were conducted in the months of June and July each year. We included households with an adult woman. We randomly sampled 400 households in 2008, 2009 and 2010, 1170 households in 2011 and 1200 households in 2012, 2013, 2014 and 2015. We initially powered the sample to estimate the percentage of children aged 0-59 months with a fever in the 2 weeks prior to the survey who received an effective antimalarial within 24 hours of symptom onset, in a population of 56000 , with a $95 \%$ confidence level, a $5 \%$ margin of error and a conservative $50 \%$ response distribution.

A two-stage cluster sampling methodology, with probability of selection proportional to population size, was used to achieve a representative sample of the population in the intervention area. In the first stage of sampling, we generated 40 (2008-2010) or 65 (2011-2015) random non-overlapping latitude-longitude coordinates with $100 \mathrm{~m}$ radial buffers within the area of the intervention. As a measure of population density, we counted the number of structures within each cluster, using less than 6-month-old satellite imagery. In the second stage of sampling, interviewers selected households by arriving at the centre of the cluster and spinning a pen to identify a random direction to determine the initial direction to follow. They then visited every second household along that trajectory and chose a predetermined number of households proportional to the population density in that cluster. Within each household, a Kish $\operatorname{grid}^{61}$ for random participant selection was used to randomly select one respondent, a female aged 16 years or over. In accordance with Malian law, married women younger than age 16 were also considered adults and included in the survey. The survey, which included questions about household demographic characteristics, fever in children under five and birth histories, was adapted primarily from Mali's national Demographic and Health Survey, using existing validated tools created by ICF International and PSI. ${ }^{62}$ Interviewers were hired for the sole purpose of survey administration and were not members of the communities they surveyed.

Demographic characteristics of respondents were similar across study years (online supplementary table $1 \mathrm{~A}, \mathrm{~B})$.

\section{PATIENT VISITS AND PROGRAMME OUTPUTS}

Patient data were collected through patient encounter forms completed by the government primary health centre staff and CHWs. A data entry professional based at the government primary healthcare centre maintained an electronic database that tracked the number of patient encounters in which user fees were removed. The number of sick patient visits per month was tracked by primary health centre staff and made available by the Ministry of Health District Health Information Systems office. At each patient encounter, CHWs recorded when the encounter began and when symptom onset began, from which we calculated the symptom-to-care time and the percentage of CHW patients aged 0-59 months who received care within 24 hours of symptom onset. This clinical data collected for programmatic outputs complemented data collected separately on symptom-to-treatment time in the household survey.

In the years after the roll-out of ProCCM, documented patient visits in the home or the clinic increased 10-fold. During the period of the intervention, CHWs conducted 618877 proactive case finding home visits, and 29561 home visits for sick children 0-59 months old. In the 2015 survey, $77 \%$ of participants reported that they had received a home visit from a CHW within the past 3 years. Of those who reported receiving CHW home visits in that survey, $59 \%$ had been visited within the past month, and $77 \%$ had been visited within the past 3 months. The Yirimadio public community health centre conducted 231873 sick patient visits and follow-ups for patients of all ages, of which 106050 (46\%) had all user fees removed (figure 1).

CHWs were trained and supervised to assess each patient first for danger signs, indicating need for emergently 


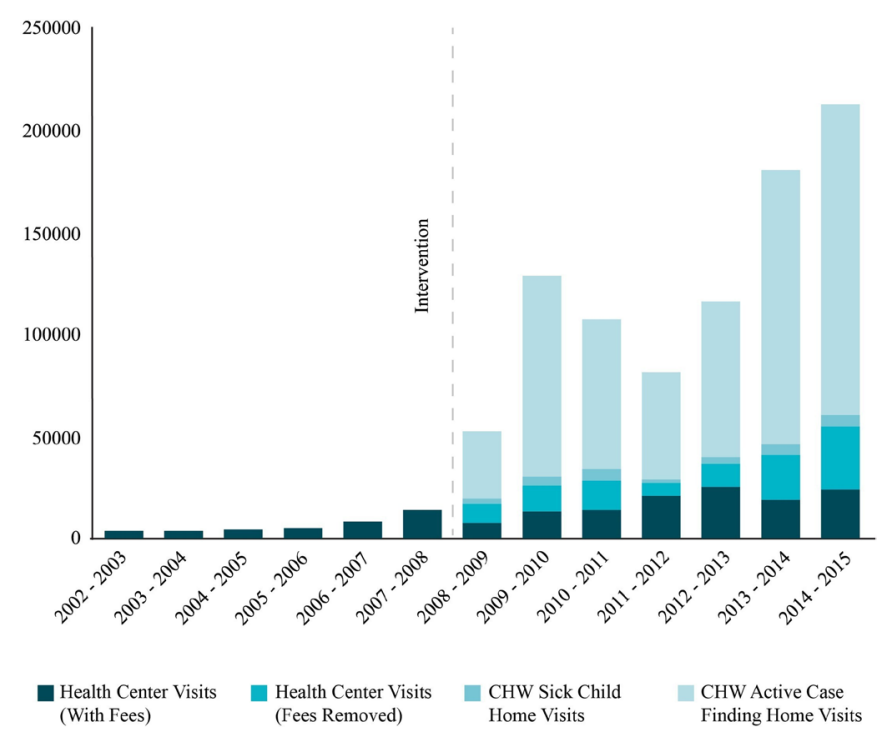

Figure 1 Number of patient-provider visits in the home and in the public primary health centre, before and after the launch of the ProCCM intervention. CHW, community health worker; ProCCM, proactive community case management.

evacuating the patient to the primary care centre, and referral signs, indicating the need to refer the patient to the primary care centre because of a condition beyond the CHW's scope of practice. Of patients evaluated by CHWs aged 0-59 months, 28\% had a danger or referral sign requiring treatment at the primary care facility, and $17 \%$ had a positive malaria rapid antigen diagnostic test. Test positivity ranged from a low of $6 \%$ in the dry season months (February to June) to a peak of $42 \%$ in the rainy season months (October and November). Neither the design of the survey tool nor the data quality from the primary care facility permits a breakdown of morbidity or mortality by cause among patients treated. The percentage of CHW patients aged 0-59 months reached within 24 hours of symptom onset was $25 \%$ in year 1 and $62 \%$ in year 7 (online supplementary figure 1).

The intervention costed approximately US $\$ 8$ per person served per year (ranging from approximately
US\$6 to US\$13 per person per year). As the intervention provided care to patients of all ages, aimed at full population coverage, cost per person was calculated by dividing total intervention costs by the estimated total population of the catchment area. This cost, when added to Mali's existing government spending on healthcare, would still be well within the range of what governments in the region are spending on healthcare, and consistent with Abuja Declaration targets. ${ }^{63}$ A detailed study of intervention costing and cost-effectiveness is currently in process.

\section{FEBRILE ILLNESS AND EARLY TREATMENT INITIATION}

We calculated the proportion of children in the household under five (both biological and non-biological children of respondents) who were reported to be febrile in the 2 weeks prior to the survey and the proportion of febrile children under five who received an effective antimalarial treatment within 24 hours of symptom onset. We estimated prevalence using SAS (v9.4) accounting for clustering and strata with surveyfreq command. The Surveylogistic command was used to predict and compare the prevalence and/or ORs for the covariates in each logistic regression model, adjusted for clustering and sampling weight. The 'study year' (2008 to 2015) was treated as a categorical variable with 2008 as the reference year. Two other covariates, 'having nets in household' and 'having attended any school,' were included in the models when appropriate and/or significant to adjust the logistic models. The model fit the data well, and the convergence criteria were satisfied.

The prevalence of febrile illness among children under 5 years in the 2 weeks prior to the survey decreased significantly from $39.7 \%$ in 2008 to $22.6 \%$ in 2015 (OR 0.448, $\mathrm{P}<0.0001$, table 1$)$, a relative reduction of $55.2 \%$. The lowest prevalence was observed in $2014(15.7 \%)$, a relative reduction of $71.5 \%$ from 2008 (OR $0.285, \mathrm{P}=0.0002$, table 1).

Among febrile children under 5 years, the percentage who received effective antimalarial treatment within

Table 1 Percentage and estimated OR of febrile illness among children under 5 years in the 2 weeks prior to the survey, from 2009 to 2015, after the introduction of the ProCCM intervention, compared with the baseline figure in 2008 (survey logistic regression model)

\begin{tabular}{llllllll}
\hline \multirow{2}{*}{$\begin{array}{l}\text { Study } \\
\text { year }\end{array}$} & $\mathbf{n}$ & & & & \multicolumn{3}{l}{ Survey logistic regression model } \\
\cline { 7 - 8 } \cline { 6 - 7 } & Missing & Count & Percentage & OR & $\mathbf{9 5 \%} \mathbf{C l}$ & P value \\
\hline 2008 & 292 & 0 & 116 & 39.7 & 1.00 & & Ref \\
\hline 2009 & 426 & 0 & 91 & 21.4 & 0.392 & 0.263 to 0.583 & $<0.0001$ \\
\hline 2011 & 463 & 0 & 129 & 27.9 & 0.591 & 0.405 to 0.862 & 0.0063 \\
\hline 2012 & 1317 & 0 & 299 & 22.7 & 0.372 & 0.268 to 0.517 & $<0.0001$ \\
\hline 2013 & 1056 & 0 & 317 & 33.2 & 0.762 & 0.550 to 1.055 & 0.1015 \\
\hline 2014 & 1056 & 0 & 321 & 30.4 & 0.665 & 0.484 to 0.912 & 0.0113 \\
\hline 2015 & 985 & 0 & 166 & 15.7 & 0.285 & 0.204 to 0.400 & $<0.0001$ \\
\hline
\end{tabular}

ProCCM, proactive community case management. 
Table 2 Percentage and estimated OR of receiving effective antimalarial treatment within 24 hours among febrile children under 5 years of age in the 2 weeks prior to the survey, from 2009 to 2015 , after the introduction of the ProCCM intervention, compared with baseline in 2008

\begin{tabular}{|c|c|c|c|c|c|c|c|}
\hline \multirow[b]{2}{*}{ Study year } & \multirow[b]{2}{*}{$\mathbf{n}$} & \multirow[b]{2}{*}{ Missing } & \multirow[b]{2}{*}{ Count } & \multirow[b]{2}{*}{ Percentage } & \multicolumn{3}{|c|}{ Survey logistic regression model } \\
\hline & & & & & OR & $95 \% \mathrm{Cl}$ & $P$ value \\
\hline 2008 & 116 & 0 & 17 & 14.655 & 1.00 & & Ref \\
\hline 2010 & 129 & 0 & 58 & 44.961 & 4.756 & 2.530 to 8.940 & $<0.0001$ \\
\hline 2011 & 299 & 0 & 91 & 30.435 & 2.395 & 1.324 to 4.333 & 0.0039 \\
\hline 2013 & 321 & 5 & 120 & 31.975 & 3.620 & 2.048 to 6.398 & $<0.0001$ \\
\hline 2014 & 166 & 0 & 108 & 65.060 & 10.842 & 5.850 to 20.091 & $<0.0001$ \\
\hline 2015 & 223 & 2 & 78 & 35.294 & 3.198 & 1.749 to 5.847 & 0.0002 \\
\hline
\end{tabular}

ProCCM, proactive community case management.

24 hours of symptom onset more than doubled during the study period, from $14.7 \%$ in 2008 to $35.3 \%$ in 2015 (OR 3.198, $\mathrm{P}<0.0001$, table 2 ). The percentage who received early antimalarial treatment peaked in 2014 at $65.1 \%$, with the odds of early treatment almost 11 times the odds in 2008 (OR 10.842, $\mathrm{P}<0.0001$ ). During the same period, national rates of early effective antimalarial treatment remained flat and low: 15\% in 2006 and 15\% in 2012-2013 Demographic and Health Survey studies. ${ }^{64}$ This is consistent with low rates of access to artemisinin-based combination therapy across sub-Saharan Africa. ${ }^{65}$

The respondent's education status was significantly associated with effective early treatment of febrile illness among children under 5 years. Effective antimalarial treatment within 24 hours of symptom onset increased by $49 \%$ if the female respondent had attended any school compared with no school (OR $1.49, \mathrm{P}<0.01$ ). However, education status was not significant in the model when the interaction terms were included, and thus, the model with year only was used to estimate all ORs (table 2).

An important limitation to note is that the primary indicator of early effective antimalarial treatment measured treatment rates among all children with fever in 2 weeks prior to the survey rather than among all children with test-confirmed malaria. This was due to a lack of highquality data on malaria diagnostic use for the full duration of the study.

A $55 \%$ relative decrease occurred in the percentage of children 0-59 months with all-cause febrile illness. The documented increases in early treatment across the population likely shortened infection duration and may have reduced transmission of malaria, pneumonia and diarrhoeal disease. Treating cases of malaria within 24 hours of symptom onset, for example, could have eradicated Plasmodium falciparum malaria infections before the transmissible gametocyte form emerged. ${ }^{66} 67$ In Senegal, weekly proactive case detection was associated with a significant reduction in the odds of symptomatic malaria infection. ${ }^{68}$

\section{UNDER-FIVE MORTALITY}

Under-five mortality rates for all births reported by respondents in the 5 years prior to each survey were computed and compared. We used a Cox proportional hazard survival regression to compare risk of death before the age of 5 years across surveys, using survey year (2008 to 2015) as the explanatory variable and the 2008 survey as the reference.

The multiple imputation procedures MI and MIANALYZE in SAS, based on stochastic regression and maximum likelihood methods for the fully conditional specification, were used to estimate missing values for age of death (in months), month of death and year of death. After multiple imputation of missing values, the participation time of each child under 5 years within a year was estimated for the Cox proportional hazard survival regression. We accounted for clustering of observations by sampling survey area. Children under five still alive at the time of survey were right censored. All the criteria were met for the proportional hazard model, and the results were analysed using the multiply imputed datasets.

In online supplementary table 2, we present the missing patterns of the dataset with the percentage of complete or incomplete information for each variable to be imputed. The minimum relative efficiency of the imputed dataset was 0.999985 , meaning that $99.9985 \%$ of the true parameter values were estimated for the variables. Also, the maximum value of the fraction missing info (FMI) for the variables was 0.000763 , meaning only $0.076 \%$ of sampling variance is attributable to missing data.

Figure 2 compares the rate of under-five mortality in the study area to national under-five mortality rates in Mali overall ${ }^{69}$ and in urban areas of Mali ${ }^{64}$ in particular. Under-five mortality rate was estimated at 154 deaths per 1000 live births in 2008 before the ProCCM intervention and at 7 deaths per 1000 live births in 2015, 7 years after intervention launch (HR $0.039 \mathrm{p}<0.0001$, table 3). In each of the 6 years since 2010, the area of intervention maintained a rate of under-five mortality equal to or less 


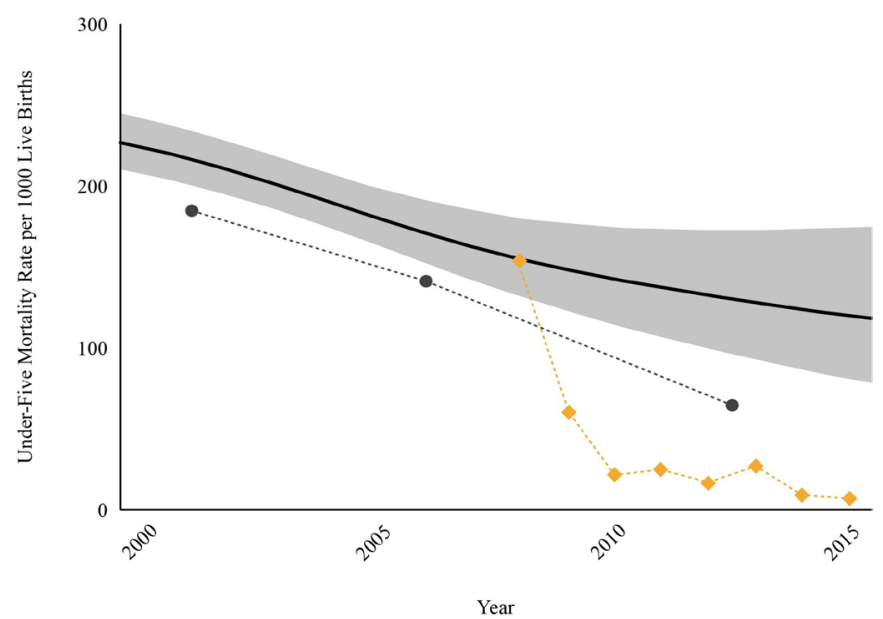

- National Estimates, UN Urban Estimates, DHS Mali $\quad$ Yirimadio Estimate

Figure 2 Under-five mortality rates before and during the ProCCM intervention, in the area of the intervention (orange diamonds), in urban areas of Mali (black circles) and nationally in Mali (black line). DHS, Demographic and Health Survey; ProCCM, proactive community case management; UN, United Nations.

than 28/1000. During the same period, Mali's estimated national under-five mortality rate was $148 / 1000$ in 2008 and at least 114/1000 through 2015 (United Nations Interagency Group for Child Mortality Estimation). ${ }^{55} 69$ The under-five mortality rate in Mali's 2012-2013 Demographic and Health Survey was 95/1000 (though this survey covered only southern Mali). ${ }^{64}$

These findings, while remarkable, are subject to several important limitations. First, this interrupted time series study design had no control group, and therefore, no causal inference can be made attributing the documented changes in health outcomes to the ProCCM intervention, to specific elements of ProCCM or to concurrent interventions addressing the social determinants of health. We cannot draw conclusions as to whether the differences in under-five mortality were due to demographic shifts, other national trends or other factors. The study did not collect information regarding household wealth, parasite prevalence or vaccination coverage. We cannot rule out the possibility that, in the context of rapid urbanisation, the population make-up in the study area changed in some systematic way that contributed to the study findings. However, the sociodemographic indicators collected in the study remained similar over the study period, providing no indication of an overall demographic shift in the population.

The study only conducted one preintervention survey, leaving open the possibility that the baseline rate recorded was an abnormal year. However, that baseline rate is consistent with national and urban rates at the time. The study also involved only one periurban site, limiting the conclusions that can be made regarding the study's generalizability.

The rate of decline in under-five mortality rate in the first survey years is faster than would be expected given that rates were based on all births in the 5 years prior to each survey. Four factors may have contributed here: (1) small sample size in the first three survey years resulted in wider CIs with the possibility of an overestimate of the baseline rate or an underestimate of the rate in 2009 or 2010; (2) as shown in figure 2, Mali's under-five mortality rate was already declining during this period, and likely contributed an uneven distribution of child deaths across the study years, though given the recorded national changes in under-five mortality, the contribution of this factor was likely modest; (3) recall error could have led respondents to under-report deaths occurring more than a year prior to the survey or (4) demographic changes could have occurred in the study population that were not captured by the sociodemographic indicators measured in the survey.

The original sample size of 400 households of the first three surveys (2008 to 2010) was powered for the outcome of early treatment with an effective antimalarial. Although the sample size was not powered for underfive mortality rate, we, nevertheless, observed significant differences in under-five child mortality in each year

Table 3 Mortality rate per 1000 live births, $\mathrm{HR}$ and $95 \% \mathrm{Cl}$ in children under 5 years old (survey proportional hazard model) from 2008 to 2015, with 2008 as the reference

\begin{tabular}{|c|c|c|c|c|c|c|}
\hline Year & No of live births & No of deaths & $\begin{array}{l}\text { Under-five } \\
\text { mortality per } \\
1000 \text { live births }\end{array}$ & HR & $95 \% \mathrm{CI} \mathrm{HR}$ & $P$ value \\
\hline 2008 & 330 & 39 & 154 & 1.000 & & Ref \\
\hline 2009 & 439 & 19 & 60 & 0.368 & 0.210 to 0.645 & 0.0005 \\
\hline 2010 & 512 & 8 & 21 & 0.130 & 0.060 to 0.278 & $<0.0001$ \\
\hline 2011 & 1423 & 26 & 25 & 0.153 & 0.092 to 0.254 & $<0.0001$ \\
\hline 2012 & 880 & 10 & 15 & 0.093 & 0.047 to 0.187 & $<0.0001$ \\
\hline 2013 & 1161 & 24 & 28 & 0.169 & 0.101 to 0.283 & $<0.0001$ \\
\hline 2014 & 1050 & 7 & 9 & 0.053 & 0.024 to 0.116 & $<0.0001$ \\
\hline 2015 & 1023 & 5 & 7 & 0.039 & 0.013 to 0.116 & $<0.0001$ \\
\hline
\end{tabular}


over the 7-year follow-up with respect to baseline. The slight differences in under-five mortality rate for years 2008 through 2011 reported here compared with the rates published in 2013 are attributable to the fact that all imputations were rerun for this follow-up analysis, and results differ slightly each time the multiple imputations procedure is run.

We tested whether bed net ownership was a significant confounder, using a Cox proportional hazard survival regression for under-five mortality rate, and found that bed net ownership changes in this study were not significant $(\mathrm{P}=0.88)$ in the model predicting under-five mortality rate.

Taking into account these important limitations, there is no strong evidence that confounders played a substantial role in these dramatic results. In this context, roll-out of ProCCM is the most probable explanation for the findings in this intervention.

Given that under-five mortality rates are driven by curable, time-sensitive illnesses, the documented increase in early access to care provides one possible explanation for sustained low rates of under-five mortality reported here. ProCCM is designed to remove financial, geographical, gender and infrastructural barriers that reduce and delay access to care and ultimately reduce underfive mortality through two primary mechanisms. First, increased access to earlier effective treatment can directly reduce the likelihood of progression to severe illness and death. Through proactive case detection, in-home diagnosis and treatment and rapid evacuation of patients with danger signs to the primary care facility, CHWs aimed to ensure early treatment in the home or the clinic for the leading causes of under-five mortality: neonatal illness, malaria, pneumonia and diarrhoea. While the study design does not permit an analysis of cause of death before and during the ProCCM intervention, it is reasonable to hypothesise that rapid access to evidence-based treatment could have reduced mortality for all of these conditions, including neonatal illnesses responsible for $36 \%$ of under-five deaths in Mali. ${ }^{64}$ Second, in the case of malaria, diarrhoeal disease and pneumonia, earlier treatment can reduce the time period during which a patient can infect others.

\section{CONCLUSION}

In 2015, the United Nations committed to the Sustainable Development Goals, which include each country achieving universal health coverage and attaining an under-five mortality rate of no more than $25 / 1000$ by 2030. As part of the efforts to achieve these goals in improving access to care and child survival, 28 countries in sub-Saharan Africa have deployed iCCM by CHWs. ${ }^{37}$ While multiple studies have shown that this CHW-led strategy can reduce newborn and under-five mortality, efforts to scale up iCCM have been met with mixed results, raising questions about how CHWs can be deployed most effectively, particularly in rapidly growing urban areas.
After the roll-out of ProCCM, the areas studied here have sustained a rate of under-five mortality lower than that of any country in sub-Saharan Africa for the past 6 years.

Amid rapid urbanisation throughout the region, health systems will need to overcome the multiplicity of barriers urban patients face to accessing timely care in order to stop preventable child deaths. Rapid population growth during the intervention also necessitated recruitment of additional CHWs, expansion of infrastructure capacity and annual adjustment of medication and supply needs.

Further research is urgently needed on ProCCM and its core strategies, to determine their respective role in improving morbidity and mortality. A randomised controlled trial of ProCCM's proactive case detection approach is currently underway across 137 cluster sites, designed to address some of these limitations (ClinicalTrials.gov NCT02694055).

Acknowledgements We are grateful to Brian Greenwood, Sidney Atwood, Dana Thomson, Megan Murray, lan Alley, Jessica Beckerman, Hamed Diallo, Boubacar Traoré, Klenon Traoré, Paul Farmer, Joia Mukherjee, Alan Zaslavsky, Earl Francis Cook, Cathy Yoon, Mary Kay Smith Fawzi, Grant Dorsey, Phil Rosenthal and Mamary Kone for their guidance regarding the study. We are grateful to Aarti Shah for preparing the study figures and tables and to Rebecca Skala and Julia Berman for their editing. We thank Molly Lauria, Michaela Kupfer, Mary Virginia Thur, Rebecca Kosowicz, Amber Gladney and Rebecca Palmer for their training of the survey team and coordination of survey administration. We thank the Yirimadio Community Health Center clinical team, the Malian Ministry of Health, the communities of Yirimadio and the operational teams of Tostan, Muso and Partners In Health for their collaboration.

Contributors ADJ, DD and FT were involved in the design of the study. KK, BP, DD, FT and IC led the data collection. OT led data cleaning and data analysis. ADJ, CW and $\mathrm{OT}$ wrote the manuscript. All authors provided input in the revision and final approval of the manuscript and met the ICMJE authorship conditions.

Funding Funds from the Child Relief International Foundation supported this research.

Competing interests None declared.

Patient consent Participant/guardian consent obtained.

Ethics approval This study was reviewed and approved by the ethical committee of the Faculty of Medicine and Odontostomatology of the University of Bamako, Mali, reference 2016/40/CE/FMPOS. The study was also reviewed by the University of California, San Francisco, Human Research Protection Program Committee on Human Research and approved via exempt certification as posing minimal risk to the population being studied, reference nos 10-02198, 004193 and 070270.

Provenance and peer review Not commissioned; externally peer reviewed.

Data sharing statement The anonymised data sets used for this analysis are available upon request with the corresponding author. If the reader requires any further information, they are welcome to contact the corresponding author.

Open Access This is an Open Access article distributed in accordance with the Creative Commons Attribution Non Commercial (CC BY-NC 4.0) license, which permits others to distribute, remix, adapt, build upon this work non-commercially, and license their derivative works on different terms, provided the original work is properly cited and the use is non-commercial. See: http://creativecommons.org/ licenses/by-nc/4.0/

(c) Article author(s) (or their employer(s) unless otherwise stated in the text of the article) 2018. All rights reserved. No commercial use is permitted unless otherwise expressly granted.

\section{REFERENCES}

1. United Nations DoEaSA, Population Division World Urbanization Prospects: The 2014 Revision, Highlights. 2014. 
2. UN-Habitat. Urbanization and development: emerging futures,. World cities report, 2016. http://wcr.unhabitat.org/wp-content/ uploads/2017/02/WCR-2016-Full-Report.pdf (accessed 20 Oct 2017).

3. Darmstadt GL, Bhutta ZA, Cousens S, et al. Evidence-based, costeffective interventions: how many newborn babies can we save? Lancet 2005;365:977-88.

4. Lassi ZS, Middleton PF, Crowther C, et al. Interventions to Improve Neonatal Health and Later Survival: An Overview of Systematic Reviews. EBioMedicine 2015;2:985-1000.

5. Bhutta ZA, Das JK, Walker N, et al. Interventions to address deaths from childhood pneumonia and diarrhoea equitably: what works and at what cost? Lancet 2013;381:1417-29.

6. Liu L, Oza S, Hogan D, et al. Global, regional, and national causes of child mortality in 2000-13, with projections to inform post-2015 priorities: an updated systematic analysis. Lancet 2015;385:430-40.

7. Department RBM. Scaling up home-based management of malaria. From research to implementation. Geneva: World Health Organization, 2004.

8. Taylor TE. Treatment of severe malaria. Daily J, ed. UpToDate. Waltham, MA: UpToDate, 2017.

9. Qamar FN, Zaman U, Quadri F, et al. Predictors of diarrheal mortality and patterns of caregiver health seeking behavior in in Karachi, Pakistan. J Glob Health 2016;6:020406.

10. Reyes H, Perez-Cuevas R, Salmeron J, et al. Infant mortality due to acute respiratory infections: the influence of primary care processes. Health Policy Plan 1997;12:214-23.

11. Källander K, Hildenwall H, Waiswa $\mathrm{P}$, et al. Delayed care seeking for fatal pneumonia in children aged under five years in Uganda: a caseseries study. Bull World Health Organ 2008;86:332-8.

12. Ersdal HL, Mduma E, Svensen E, et al. Early initiation of basic resuscitation interventions including face mask ventilation may reduce birth asphyxia related mortality in low-income countries: a prospective descriptive observational study. Resuscitation 2012;83:869-73.

13. Lee AC, Cousens S, Wall SN, et al. Neonatal resuscitation and immediate newborn assessment and stimulation for the prevention of neonatal deaths: a systematic review, meta-analysis and Delphi estimation of mortality effect. BMC Public Health 2011;11(Suppl 3):S12.

14. Waiswa P, Kallander K, Peterson S, et al. Using the three delays model to understand why newborn babies die in eastern Uganda. Trop Med Int Health 2010;15:964-72.

15. Haines A, Sanders D, Lehmann U, et al. Achieving child survival goals: potential contribution of community health workers. Lancet 2007;369:2121-31.

16. James C, Morris SS, Keith R, et al. Impact on child mortality of removing user fees: simulation model. BMJ 2005;331:747-9.

17. WHO. Health systems financing: the path to universal coverage. The world health report: World Health Organization, 2010.

18. Jacobs $B$, Price N. The impact of the introduction of user fees at a district hospital in Cambodia. Health Policy Plan 2004;19:310-21.

19. Nabyonga J, Desmet M, Karamagi $\mathrm{H}$, et al. Abolition of costsharing is pro-poor: evidence from Uganda. Health Policy Plan 2005;20:100-8.

20. Yates R. Universal health care and the removal of user fees. Lancet 2009;373:2078-81.

21. Johnson A, Goss A, Beckerman J, et al. Hidden costs: the direct and indirect impact of user fees on access to malaria treatment and primary care in Mali. Soc Sci Med 2012;75:1786-92.

22. Adjiwanou V, LeGrand T. Gender inequality and the use of maternal healthcare services in rural sub-Saharan Africa. Health Place 2014;29:67-78.

23. Pratley P. Associations between quantitative measures of women's empowerment and access to care and health status for mothers and their children: A systematic review of evidence from the developing world. Soc Sci Med 2016;169:119-31.

24. Kiwanuka SN, Ekirapa EK, Peterson S, et al. Access to and utilisation of health services for the poor in Uganda: a systematic review of available evidence. Trans $R$ Soc Trop Med Hyg 2008;102:1067-74.

25. Balarajan Y, Selvaraj S, Subramanian SV. Health care and equity in India. Lancet 2011;377:505-15.

26. Peters DH, Garg A, Bloom G, et al. Poverty and access to health care in developing countries. Ann N Y Acad Sci 2008;1136:161-71.

27. WHO U. Who/Unicef. WHO / UNICEF Joint Statement: Integrated Community Case Management: An equity-focused strategy to improve access to essential treatment services for children. 2012 http://www.who.int/maternal_child_adolescent/documents/ statement child services access_whounicef.pdf (cited 29 Sep 2017).
28. Das JK, Lassi ZS, Salam RA, et al. Effect of community based interventions on childhood diarrhea and pneumonia: uptake of treatment modalities and impact on mortality. BMC Public Health 2013;13(Suppl 3):S29

29. Freeman PA, Schleiff M, Sacks E, et al. Comprehensive review of the evidence regarding the effectiveness of community-based primary health care in improving maternal, neonatal and child health: 4 . child health findings. J Glob Health 2017;7:010904.

30. Kidane G, Morrow RH. Teaching mothers to provide home treatment of malaria in Tigray, Ethiopia: a randomised trial. Lancet 2000;356:550-5.

31. Munos MK, Walker CL, Black RE. The effect of oral rehydration solution and recommended home fluids on diarrhoea mortality. Int $J$ Epidemiol 2010;39(Suppl 1):i75-i87.

32. Sazawal S, Black RE, Pneumonia Case Management Trials Group. Effect of pneumonia case management on mortality in neonates, infants, and preschool children: a meta-analysis of communitybased trials. Lancet Infect Dis 2003;3:547-56.

33. Walker CL, Black RE. Zinc for the treatment of diarrhoea: effect on diarrhoea morbidity, mortality and incidence of future episodes. Int $J$ Epidemiol 2010;39(Suppl 1):i63-i69.

34. Theodoratou E, Al-Jilaihawi S, Woodward F, et al. The effect of case management on childhood pneumonia mortality in developing countries. Int J Epidemiol 2010;39(Suppl 1):i155-i171.

35. Black RE, Taylor CE, Arole S, et al. Comprehensive review of the evidence regarding the effectiveness of community-based primary health care in improving maternal, neonatal and child health: 8. summary and recommendations of the Expert Panel. J Glob Health 2017;7:010908

36. Sacks E, Freeman PA, Sakyi K, et al. Comprehensive review of the evidence regarding the effectiveness of community-based primary health care in improving maternal, neonatal and child health: 3. neonatal health findings. J Glob Health 2017;7:010903.

37. Rasanathan $\mathrm{K}$, Muñiz M, Bakshi S, et al. Community case management of childhood illness in sub-Saharan Africa - findings from a cross-sectional survey on policy and implementation. J Glob Health 2014;4:020401.

38. Rasanathan $\mathrm{K}$, Bakshi S, Rodriguez DC, et al. Where to from here? Policy and financing of integrated community case management (iCCM) of childhood illness in sub-Saharan Africa. $J$ Glob Health 2014;4:020304.

39. Perry HB, Sacks E, Schleiff M, et al. Comprehensive review of the evidence regarding the effectiveness of community-based primary health care in improving maternal, neonatal and child health: 6 . strategies used by effective projects. J Glob Health 2017;7:010906.

40. Druetz T, Siekmans K, Goossens S, et al. The community case management of pneumonia in Africa: a review of the evidence. Health Policy Plan 2015;30:253-66.

41. Rutebemberwa E, Kadobera D, Katureebe S, et al. Use of community health workers for management of malaria and pneumonia in urban and rural areas in eastern Uganda. Am J Trop Med Hyg 2012;87:30-5.

42. Druetz T, Ridde V, Kouanda S, et al. Utilization of community health workers for malaria treatment: results from a three-year panel study in the districts of Kaya and Zorgho, Burkina Faso. Malar J 2015:14:71.

43. Amouzou A, Hazel E, Shaw B, et al. Effects of the integrated Community Case Management of Childhood Illness Strategy on Child Mortality in Ethiopia: A Cluster Randomized Trial. Am J Trop Med Hyg 2016;94:596-604

44. Amouzou A, Kanyuka M, Hazel E, et al. Independent evaluation of the integrated community case management of childhood Illness strategy in Malawi using a national evaluation platform design. Am J Trop Med Hyg 2016;94:1434-5.

45. Munos M, Guiella G, Roberton T, et al. Independent evaluation of the rapid scale-up program to reduce under-five mortality in Burkina Faso. Am J Trop Med Hyg 2016:94:584-95.

46. Hazel E, Bryce J, IIP-JHU iCCM Evaluation Working Group. On bathwater, babies, and designing programs for impact: evaluations of the integrated community case management strategy in Burkina Faso, Ethiopia, and Malawi. Am J Trop Med Hyg 2016;94:568-70.

47. Ghei K, Agarwal S, Subramanyam MA, et al. Association between child immunization and availability of health infrastructure in slums in India. Arch Pediatr Adolesc Med 2010;164:243-9.

48. Babirye JN, Engebretsen IM, Rutebemberwa E, et al. Urban settings do not ensure access to services: findings from the immunisation programme in Kampala Uganda. BMC Health Serv Res 2014;14:111.

49. Tomlinson M, Doherty T, ljumba P, et al. Goodstart: a cluster randomised effectiveness trial of an integrated, community-based package for maternal and newborn care, with prevention of mother- 
to-child transmission of HIV in a South African township. Trop Med Int Health 2014;19:256-66.

50. Nsibande D, Doherty T, ljumba P, et al. Assessment of the uptake of neonatal and young infant referrals by community health workers to public health facilities in an urban informal settlement, KwaZuluNatal, South Africa. BMC Health Serv Res 2013;13:47.

51. Marcil L, Afsana K, Perry HB. First steps in initiating an effective maternal, neonatal, and child health program in urban slums: the BRAC Manoshi project's experience with community engagement, social mapping, and census taking in Bangladesh. $J$ Urban Health 2016;93:6-18.

52. Akweongo P, Agyei-Baffour $\mathrm{P}$, Sudhakar $\mathrm{M}$, et al. Feasibility and acceptability of ACT for the community case management of malaria in urban settings in five African sites. Malar J 2011;10:240.

53. Amuyunzu-Nyamongo M. Community case management of malaria in urban settings. A feasibility study in five African sites. UNICEF/ UNDP/World Bank/WHO Special Programme for Research and Training in Tropical Diseases. 2011.

54. Johnson AD, Thomson DR, Atwood S, et al. Assessing early access to care and child survival during a health system strengthening intervention in Mali: a repeated cross sectional survey. PLoS One 2013;8:e81304.

55. D. HI Y, Ejdemyr S, Beise J. Levels \& trends in child mortality: report 2015, estimates developed by the un inter-agency group for child mortality estimation. 2015 http://childmortality.org/files_v20/ download/IGME report 2015 child mortality final.pdf

56. Bn AJS, De Ferranti DM. the World Bank. Financing health services in developing countries: an agenda for reform. A World Bank policy study. 1988. http://documents.worldbank.org/curated/en/ 468091468137379607/Financing-health-services-in-developingcountries-an-agenda-for-reform (accessed 29 Sep 2017).

57. Beauchemin C, Bocquier P. Migration and Urbanisation in Francophone West Africa: An Overview of the Recent Empirical Evidence. Urban Stud 2004;41:2245-72.

58. MUSO Health. Available from: www.musohealth.org (accessed 29 Sep 2017).
59. Tostan. Available from: https://www.tostan.org/ (accessed 29 Sep 2017).

60. Habicht JP, Victora CG, Vaughan JP. Evaluation designs for adequacy, plausibility and probability of public health programme performance and impact. Int J Epidemiol 1999;28:10-18.

61. Kish L. A Procedure for Objective Respondent Selection within the Household. J Am Stat Assoc 1949;44:380-7.

62. DHS M. DHS model questionnaire phase 6 (2008-2013). 2008. http://www.measuredhs.com/publications/publication-dhsq6-dhsquestionnaires-and-manuals.cfm (accessed 6 Nov 2013).

63. WHO. The abuja declaration: ten years on 2011. http://www.who. int/healthsystems/publications/abuja report aug_2011.pdf?ua=1 (accessed 20 Oct 2017).

64. Cellule de Planification et de Statistique - CPS/SSDSPF/Mali INdISIM, Centre d'Études et d'Information Statistiques - INFO-STAT/Mali and ICF International. Enquête Démographique et de Santé au Mali 2012-2013. Rockville, MD, USA: CPS, INSTAT, INFO-STAT and ICF International, 2014

65. Bennett A, Bisanzio D, Yukich JO, et al. Population coverage of artemisinin-based combination treatment in children younger than 5 years with fever and Plasmodium falciparum infection in Africa, 2003-2015: a modelling study using data from national surveys. Lancet Glob Health 2017;5:e418-e427.

66. Landier J, Parker DM, Thu AM, et al. The role of early detection and treatment in malaria elimination. Malar J 2016;15:363.

67. Eichner M, Diebner HH, Molineaux L, et al. Genesis, sequestration and survival of Plasmodium falciparum gametocytes: parameter estimates from fitting a model to malariatherapy data. Trans $R$ Soc Trop Med Hyg 2001;95:497-501.

68. Linn AM, Ndiaye Y, Hennessee I, et al. Reduction in symptomatic malaria prevalence through proactive community treatment in rural Senegal. Trop Med Int Health 2015;20:1438-46.

69. (IGME) UI-aGfCME. http://childmortality.org/index.php?r=site/graph - ID=MLI_Mali (accessed 29 Sep 2017). 